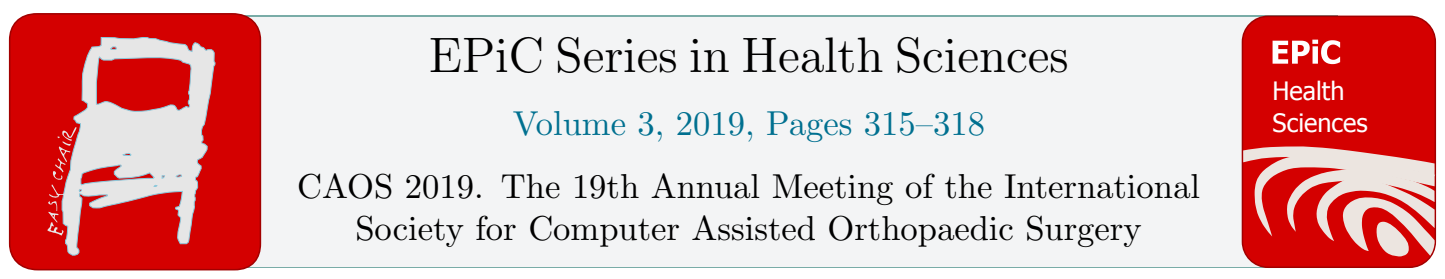

\title{
The Effect of the PCL on Kinematics as it Relates to Soft-Tissue Preservation from Robotic-Arm Assisted Surgery
}

\author{
Raga Rajaravivarma ${ }^{1}$, Sally LiArno ${ }^{1}$, Tatyana Kaverina ${ }^{1}$, Sonia Donde ${ }^{1}$, \\ Emily Sneddon ${ }^{1}$ \\ ${ }^{1}$ Stryker, Mahwah, NJ \\ raga.rajaravivarma@stryker.com
}

\begin{abstract}
The use of haptic-based robotic-arm assisted total knee arthroplasty (hRATKA) has demonstrated a decrease in soft tissue damage. Hampp et al. [4] compared the soft tissue damage in a cadaveric study between manual total knee arthroplasty (MTKA) and hRATKA and found that the hRATKA specimens had significantly less damage to the PCL than the MTKA specimens [4]. The purpose of this study is to look at the effect of the posterior cruciate ligament (PCL) on kinematics, as defined by anterior-posterior (AP) tibial translation, during stair climb. Using a 6 degree-of-freedom (6-DOF) joint motion simulator, the stair climb profile was run on four cadaveric knee specimens with the PCL intact and the PCL cut. In the PCL deficient condition, there was more posterior tibial motion than when the PCL was intact, and this was more noticeable during the high flexion portion of the profile. These findings speak to the importance of soft tissue preservation, and the development of haptic-based robotic-arm assisted TKA can help prevent soft tissue damage to the PCL.
\end{abstract}

\section{Introduction}

With computer assisted orthopaedic surgery, there are opportunities to preserve soft tissue in total knee arthroplasty (TKA). Hampp et al. compared the soft tissue damage between manual total knee arthroplasty (MTKA) and haptic-based robotic-arm assisted total knee arthroplasty (hRATKA) in a cadaveric study and found that the hRATKA specimens had significantly less damage than the MTKA specimens [4]. This was particularly true for the posterior cruciate ligament (PCL) [4]. The PCL is the primary restraint to posterior translation of the tibia and plays a role in joint compression [2]. It would therefore be expected that damage to the PCL during TKA could lead to increased laxity and thus a feeling of knee instability.

Some studies have used simulation methods to evaluate the effect of the PCL on kinematics [1,2]; however physical testing of activities of daily living can help to verify this impact. Sekiya et al. used 
stress radiography to measure the increase in posterior translation during a $200-\mathrm{N}$ posterior drawer at 90 degrees flexion with the PCL-injured specimens [3], and while this shows the PCL may affect knee stability, an active profile - rather than passive testing alone - can illustrate the extent of knee stability that is compromised with a damaged PCL. Therefore, the purpose of this study is to look at the effect of the PCL on kinematics, as defined by anterior-posterior (AP) tibial translation during stair climb.

\section{Material and Methods}

Four left cadaveric knee specimens were dissected with all soft tissue removed except the PCL, ACL, and the collateral ligaments. An intramedullary (IM) rod was implanted and fixed with bone cement in the femoral shaft. The tibia and fibula were cemented in a custom fixture with bone cement. The knee was mounted in a 6 degree-of-freedom (6-DOF) joint motion simulator, such that the epicondylar axis was aligned with the flexion axis of the hydraulic testing machine.

In order to assess the PCL in the cadaveric knees with an active profile, data from instrumented knee implants were used to derive the stair climb profile [5]. The stair climb profile was chosen as it is a common activity where the PCL is expected to play an important role to restrain the knee, particularly in high flexion [2]. The knee was first tested intact to gain input on normal stair climb kinematics for that specimen. Afterwards, the PCL was cut, and the stair climb profile was run on each specimen. The 6-DOF joint motion simulator collected the anterior-posterior tibial motion with the intact PCL and the PCL-deficient conditions, and this could be compared across all four specimens by looking at the ratio of maximum anterior and posterior motion in the cut PCL condition to the intact condition. Further analysis focusing on the higher flexion region where the PCL is engaged was conducted with one-way ANOVA to statistically determine significant differences (significance $\mathrm{p}<0.05$ ) of local peaks and valleys in the high flexion region shown in Figure 2.

\section{Results}

In all four specimens, there was more posterior motion and less anterior motion in the PCL-deficient condition. The ratio of overall maximum posterior and anterior displacement in the PCL-deficient condition to the intact PCL condition was $1.50+/-0.26$ and $0.67+/-0.19$ (Figure 1a). Though there were variations in magnitude, the same kinematic trend was seen for all four specimens, with additional posterior motion in the PCL-deficient condition (Figure 2). As expected, this was particularly notable in the high flexion region ranging from the peak at 92 degrees (P1) to the valley at 65 degrees (V1). 
The PCL-deficient condition had significantly more posterior tibial motion compared to the intact condition at $\mathrm{P} 1(\mathrm{p}=0.031)$ and $\mathrm{V} 1(\mathrm{p}=0.004)$.

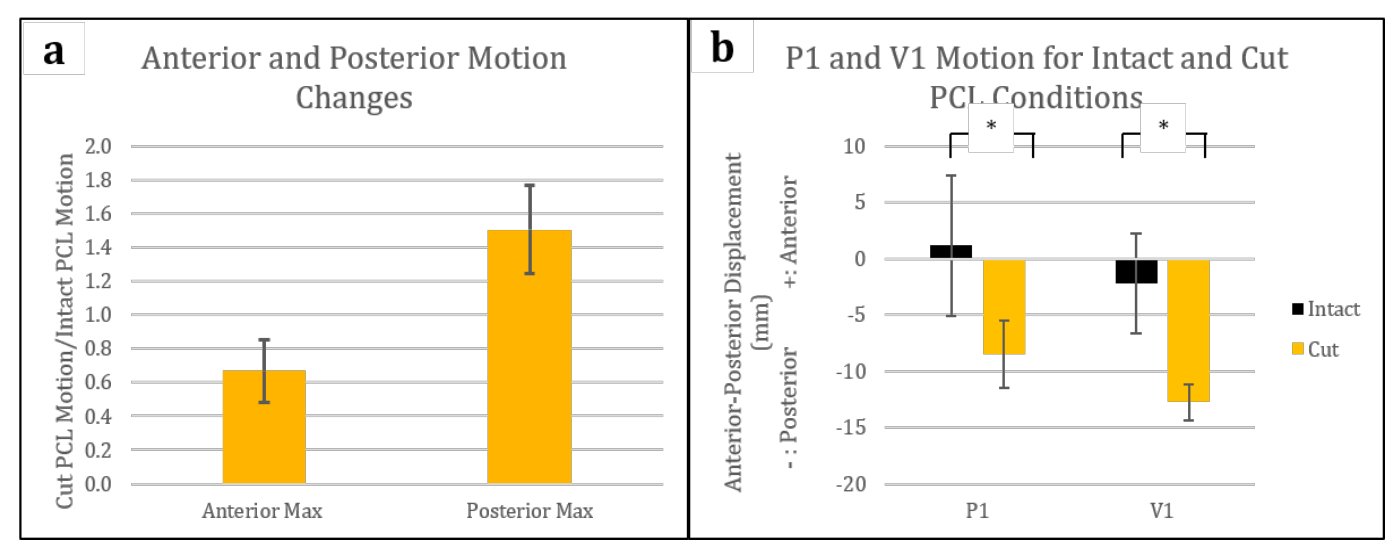

Figure 1: (a) Cut PCL to Intact PCL condition ratio of overall max anterior motion and posterior motion. (b) P1 and V1 anterior-posterior tibial location average for all 4 specimens for intact and cut PCL. * denotes significant difference.

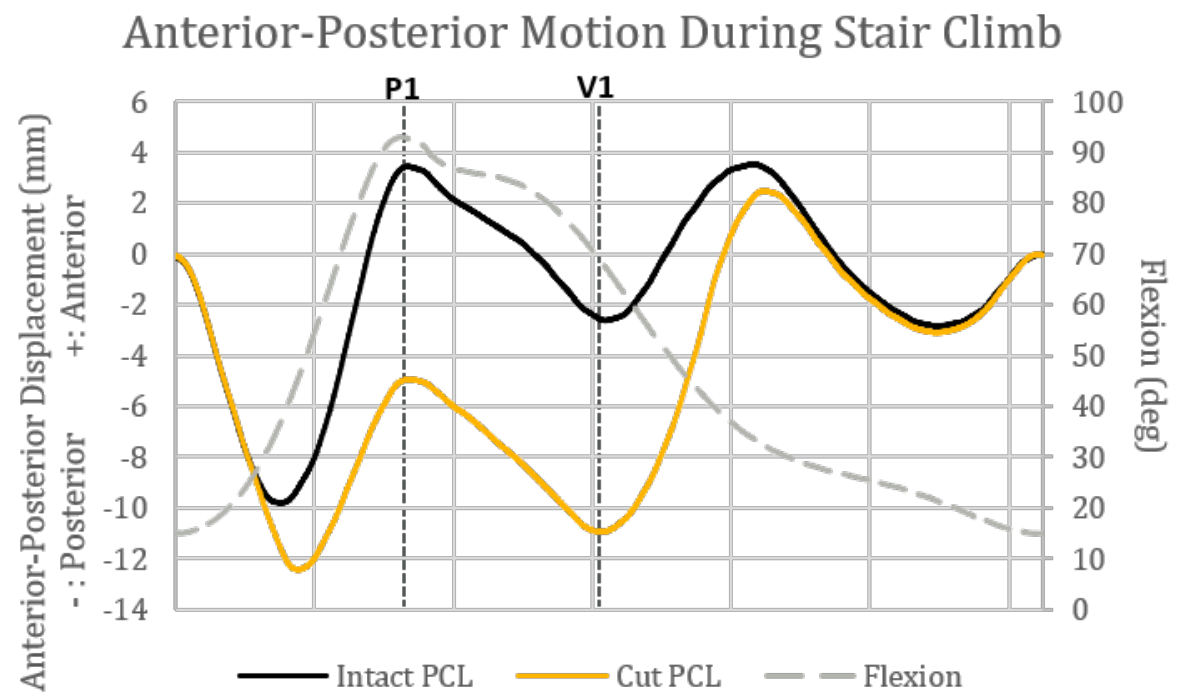

Figure 2: Anterior-posterior motion for one cycle of stair climb for Specimen 1 with the PCL intact and the PCL cut. P1 and V1 on graph shown.

\section{Discussion}

This study provided additional information about the impact of the PCL and its effect on knee stability and kinematics during an activity of daily living. During stair climb, there was an increase in posterior tibial motion with PCL-deficient specimens. Especially during the higher flexion regions of 
the profile, there was a significant posterior shift, which coincides with previous studies that examined the effect of the PCL $[1,2,3]$. This posterior shift also leads to a decrease in anterior tibial motion with PCL-deficient specimens. Surgical techniques involving the tibial cut have been explored to help protect the PCL because a functioning PCL is believed to control rollback during flexion [6,7]. During tibial cutting, PCL fibers may be detached especially when there is some posterior slope, so surgical techniques that preserve the PCL are beneficial [6, 8]. Haptic-based robotic-arm assisted TKA can better protect soft tissue like the PCL. The cadaveric study from Hampp et al. looking at soft tissue preservation in MTKA and hRATKA noted the stereotactic boundary constraint on the sawblade may help prevent soft tissue damage [4]. While many other robotic systems use cutting blocks or similar methods, new TKA robotic system applications that are haptic-based - such as the application used in Hampp et al. [4] - help protect the PCL. Learning more about the effect of the PCL on knee kinematics highlights the advantages of using hRATKA to preserve physiological motion.

\section{References}

[1] Kang, K., Koh, Y., Jung, M., Nam, J., Son, J., \& Lee, Y. et al. (2017). The effects of posterior cruciate ligament deficiency on posterolateral corner structures under gait- and squat-loading conditions. Bone \& Joint Research, 6(1), 31-42. doi: 10.1302/2046-3758.61.bjr-2016-0184.r1

[2] Shoifi Abubakar, M., Kuriyama, S., Ito, H., Ishikawa, M., Furu, M., \& Tanaka, Y. et al. (2016). Influence of Posterior Cruciate Ligament Tension on Knee Kinematics and Kinetics. The Journal Of Knee Surgery, 29(08), 684-689. doi: 10.1055/s-0036-1571803

[3] Sekiya, J., Whiddon, D., Zehms, C., \& Miller, M. (2008). A Clinically Relevant Assessment of Posterior Cruciate Ligament and Posterolateral Corner Injuries. The Journal Of Bone And Joint Surgery-American Volume, 90(8), 1621-1627. doi: 10.2106/jbjs.g.01365

[4] Hampp, E., Yanoso-Scholl, L., Westrich, G.H., Mont, M. (2018). Less Iatrogenic Soft Tissue Damage in Robotic Assisted Haptically Guided Total Knee Arthroplasty When Compared With Manual Approach. ISTA 2018, London, UK.

[5] D’Lima, D., Patil, S., Steklov, N., \& Colwell, C. (2011). The 2011 ABJS Nicolas Andry Award: 'Lab'-in-a-Knee: In Vivo Knee Forces, Kinematics, and Contact Analysis. Clinical Orthopaedics And Related Research ${ }^{\circledR}, 469(10), 2953-2970$. doi: 10.1007/s11999-011-1916-9

[6] Cinotti, G., Sessa, P., Amato, M., Ripani, F., \& Giannicola, G. (2015). Preserving the PCL during the tibial cut in total knee arthroplasty. Knee Surgery, Sports Traumatology, Arthroscopy, 25(8), 25942601. doi: 10.1007/s00167-015-3842-y

[7] Wood, A., Rabbani, T., Sheffer, B., Wagner, R., \& Sanchez, H. (2018). Protecting the PCL During Total Knee Arthroplasty Using a Bone Island Technique. The Journal Of Arthroplasty, 33(1), 102-106. doi: 10.1016/j.arth.2017.08.009

[8] Matziolis, G., Mehlhorn, S., Schattat, N., Diederichs, G., Hube, R., Perka, C., \& Matziolis, D. (2011). How much of the PCL is really preserved during the tibial cut?. Knee Surgery, Sports Traumatology, Arthroscopy, 20(6), 1083-1086. doi: 10.1007/s00167-011-1696-5

\footnotetext{
Stryker Content ID: GSNPS-AJA-143_19595
} 